\title{
Pervasive model, a new paradigm of computing applied to road transport systems
}

\author{
C. García, F. Alayón, J. Caraballo, R. Pérez \& G. Padrón \\ Dpto. Informática y Sistemas, \\ Universidad de Las Palmas de Gran Canaria, Spain
}

\begin{abstract}
We describe how mobile and ubiquitous computation models have been applied, with the aim of solving some technological traditional problems in the domain of public passenger road transport. Those problems are due to a weak integration of the information systems aboard. The introduced models have permitted us to solve our integration problems, and, consequently, we have managed to improve the business in a variety of domains. Particularly, the application of those models have benefited on-board mobile information systems and user information systems.

Keywords: information systems, intelligent transport systems, pervasive computing.
\end{abstract}

\section{Introduction}

From the irruption of personal computers, at the beginning of the eighties, to present time, with the development of mobile communication systems, we have assisted to the incessant progress of information technologies. This progress affects both productive business processes and leisure in modern societies. As might be expected, those technological innovations have also benefited the transport world in all its aspects-by air, sea or land, public or private, of passengers or goods. Nevertheless, those information systems that are installed on road transport vehicles have suffered a slower evolution. The main reasons of this gap are, on the one hand, the belated arrival of mobile communication services with attractive costs, and, on the other hand, the high cost of on-board equipments. However, during the last decade, this situation has changed because of the wider range of mobile communication possibilities in competition and the development of the mobile computation. 
The main aspects of the overall information system for public passenger road transport that we have developed will be defined next. Its most outstanding characteristic is the suitable integration of the mobile information subsystems aboard the vehicles. We have used for the development of our information system an innovative computing model that, although it was conceptually proposed by Mark Weiser [1] in the nineties, it is being applied nowadays to improve the existent information systems. We are talking about pervasive computation. This new paradigm [2] originates from distributed mobile computing and aims for the development of the ubiquitous computation systems. The purpose of ubiquitous computation is to offer users suitable services, regardless of their location, mobility or devices to access to the telecommunication and computing information systems in use. Finally, we would like to point out that our system is currently working in the transport company Global Salcai-Utinsa S.A. They carry more than 25 million passengers every year and have a fleet of more than 300 buses in Gran Canaria (Canary Islands, Spain).

\section{Challenges: aims and implications}

From a general point of view, the objective of our system is to manage that the road public transport company uses high quality information, by means of a convenient integration of the mobile systems on board its vehicles. By high quality information, we mean information obtained in the right moment and in the required quantity by all requesters of the transport company. By convenient integration, we mean incorporation of on-board systems into the corporative information system using the different communication infrastructures in an efficient and secure manner.

Thus, our model is fundamentally based on the information systems aboard and the data communication system. From a user point of view, our model eases the work of drivers and fleet control staff and improves the information provided to the client by the transport company. Furthermore, the availability of high quality information coming from the exploitation benefits the other fields in the company, particularly medium and long term service schedule.

We have two key elements among the available basic resources for reaching our goal: on-board systems technology and communication infrastructures. Regarding the former, the information systems aboard the road public transport company vehicles are the least technologically developed, compared with the rest of the systems that configure the overall corporative information system. This lack of development is due to several factors, mainly the existence of widespread solutions in the market based on proprietary technology, the use of robust but obsolete components and the high cost of those components. Regarding the communication infrastructures, the situation has substantially changed in the latest years. Indeed, whether we had before conventional radio infrastructures to transmit data with a very limited bandwidth, now we have global communication services based on mobile telephony. 
The achievement of our objective is nowadays partial. Indeed, first systems accomplished it to a certain extent and were basically limited to the fleet control systems, formerly based on communicating very limited information about the vehicle state as a response to a request from the control centre. Nowadays, some systems are appearing which integrate information from the invoicing system aboard by means of communication infrastructures based on mobile telephony. But those systems offered by the market, regardless of its evolution degree, even when they manage to provide the company with the information that it requires, have two serious drawbacks: first, they have a lack of flexibility and scalability and, second, their support costs are high.

Taking into account our previous description, we can restate the main objective of our system in the following way: to have at our disposal flexible and scalable mobile information systems and communication systems, permitting the transport company to obtain high quality information with allowable costs, both of deployment and of maintenance. A first consequence comes up of this statement: Our solution should be founded on elements, both hardware and software, based on products that are standard or largely accepted by the market. This asseveration is supported by the results that we have obtained in [3] and [4].

\section{Theoretical framework: mobile and ubiquitous computation}

Although it was in 1991 when Mark Weiser — at that time chief technology officer at Palo Alto with Xerox-postulated its vision of 21st century computer where he proposed the concept of ubiquitous computation, it is at present time when his ideas begin to have practical and economical viability. Some of the technological advances that are allowing it are wireless communication, global communication networks and positioning systems. Nevertheless, there is still a long way to arrive at his final objectives, expressed by him in [1]:

There is more information available at our fingertips during a walk in the woods than in any computer system, yet people find a walk among trees relaxing and computers frustrating. Machines that fit the human environment instead of forcing humans to enter theirs will make using a computer as refreshing as taking a walk in the woods.

Here a drastic change underlies in the concept of computer as we presently understand it. Nowadays we think of a computer as a machine running programs in a more or less virtual environment, in which we have to enter to carry out some task, and we leave it when we have finished this task. In the pervasive computation model, a device is the means which allow us to handle a data space, and not just a software repository to be operated by the user. Thus, an application is the means for a user to carry out a task, not software developed to obtain some performance from devices capabilities. Finally, a computation environment is a physical information space, not a virtual environment for storing and running software.

The pervasive computation paradigm gathers mobile, distributed and ubiquitous computation models. We can say that the mobile computation principle, that can be summarized by to be connected at any moment and in any place, is replaced 
in the pervasive model by to be connected always and everywhere (Saha and Mukherjee [2]). This kind of mobility is achieved by means of integration of pervasive technology basis like: interoperability, scalability and invisibility. In figure 1 we show the main components of pervasive computation.

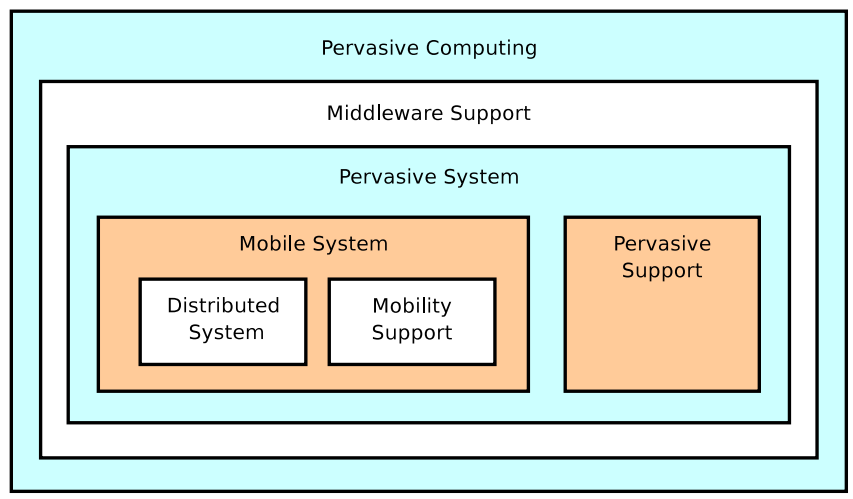

Figure 1: Pervasive computing configuration.

Figure 1 shows that pervasive computing is made of pervasive systems, which are mobile systems that work in a distributed way and are also able to autonomously integrate with its environment. To perform this integration, pervasive systems use sensitive devices, or devices capable of perceiving the context around the system. The integration between different pervasive systems is carried out through a middleware, which is responsible of handling the different communication mediums used by the systems and therefore allows pervasive applications to communicate. From a structural perspective, the characteristic components of this model are:

- Devices allowing interaction with the physical environment. They include traditional input-output devices like keyboards, mice, speakers, microphones, etc., as well as small sensitive elements like cameras, biosensors, etc. A basic role is also played by computing and communication mobile devices like mobile phones, PDAs, small laptops, etc.

- Pervasive networks aiming to integrate and communicate those devices.

- A middleware, composed of firmware and software, offering communication operations between applications running on pervasive devices. To provide these services, the middleware uses the existing networks.

From a functional perspective, the main characteristic of ubiquitous systems software is basically their capability to integrate with the physical environment. As a result of this integration they can operate in an autonomous and spontaneous way in a variety of environments. The ubiquitous systems software development must bear in mind the following principles [5]:

- Boundary principle. The difference between contexts should stem from the boundaries marking content differences. Those boundaries should not 
necessarily limit the interoperability between systems.

- Volatility principle. Ubiquitous systems should be able to manage an unpredictable number of users, devices and applications participating in a given ubiquitous context. Thus, it is necessary to specify a set of fixed working principles controlling the system execution.

By applying this model to the public passenger road transport, we can improve the company work due to the increment of the automation degree of information flows, particularly information coming from mobile devices. In our applied system, 2 million transactions are automatically performed every day, taking part in ticketing, fleet control and maintenance activities. This high degree of automation allows managers, clients and drivers to have at their disposal information updated in real-time.

\section{A pervasive system for road public transportation}

In this section we will describe the general aspects of the system. The company uses our system to control in real-time the fulfilment of the schedule: services, resources employed by those services, observance of times when the bus must arrive at bus stops. The company has also at its disposal, at real-time, the information generated by the production - the system automatically performs every day more than 2 million transfers of production records-and real-time maintenance information about equipments aboard. To achieve our general aim, we had to carry out the following requirements:

- We have configured an overall information system that integrates mobile and non-mobile systems by means of a network that hides their differences. Thus, from an integrated point of view, the distinct peculiarities of mobile and non-mobile hosts can be ignored.

- We have developed an on-board information system, flexible and scalable regarding the incorporation of new hardware and software elements. We have also strengthened the functionalities of on-board systems by adding multimedia and aware elements, aiming to improve the service security and to reply to other problems still unresolved in those environments.

- We have made available the information needed to allow the development of user information services, permitting users to obtain information about the service provided by the company, in real-time and from anywhere.

Those requirements affect the development of the on-board information systems and the communication services. We chose to develop a pervasive environment for the transport company consisting of on-board mobile systems that produce the basic corporative information and other systems that consume this information. The latter can be both a permanent element of the corporative network and a device integrated into it in a given moment, for example, the mobile phone of a user looking for some corporative information. 


\subsection{Structure}

With the pervasive model in mind, we will describe the system structure considering devices, network and middleware support. Our developments in the field of the on-board systems and the middleware are particularly outstanding because of its innovative character.

\subsubsection{Devices}

4.1.1.1 On-board system The system aboard the vehicle is composed of a computer with wireless capabilities, multimedia functionalities, GPS and a video camera connected. It supports the following functionalities: on-board invoicing system, fleet activity control and spontaneous and autonomous integration into the company information system. The on-board system also incorporates multimedia resources aiming to facilitate driver interaction and communication with passengers.

4.1.1.2 Staff query devices Query devices, such as PDAs, used by personnel like route inspectors or maintenance staff. Those devices provide them with information about the state of the exploitation or about maintenance.

4.1.1.3 Passengers query devices Query devices, like PDAs, mobile phones, etc., used by passengers to enquire about the service. Our passenger information system model goes beyond the classic model, consisting of static information points in stations and Web services to users connected to the Internet. Our system expands this model with the development of tools making easy the accessibility to the information from mobile devices like phones and PDAs.

\subsubsection{Network}

The network infrastructure consist of: the wired network to connect non-mobile enterprise hosts, the wireless local network based on 802.11 standard, used for the transactional system, the long distance wireless communication system, based on a radio trunking system, used for fleet activity control and events communication, and the mobile GPRS telephony, that permits inspectors and users to have an upto-date fleet information. Through the use of those infrastructures, our system performs autonomously and in real-time more than 2 million transactions every day, showing in a detailed way all the vehicles activity.

Our communication system is based on mailboxes as described in [6]. According to the Cao et al. model [7], the parameters characterizing a mailboxbased agents communication protocol are: mailbox migration frequency, mailboxto-agent message delivery, and migration-delivery synchronization. Next, we proceed to describe them and we explain which configuration we have chosen and justify our decision.

4.1.2.1 Mailbox migration frequency This parameter indicates how much the mailbox migrates: No migration (NM), full migration $(F M)$ or jump migration $(J M)$. Our system uses NM mode, that is, the mailbox permanently stays in the 


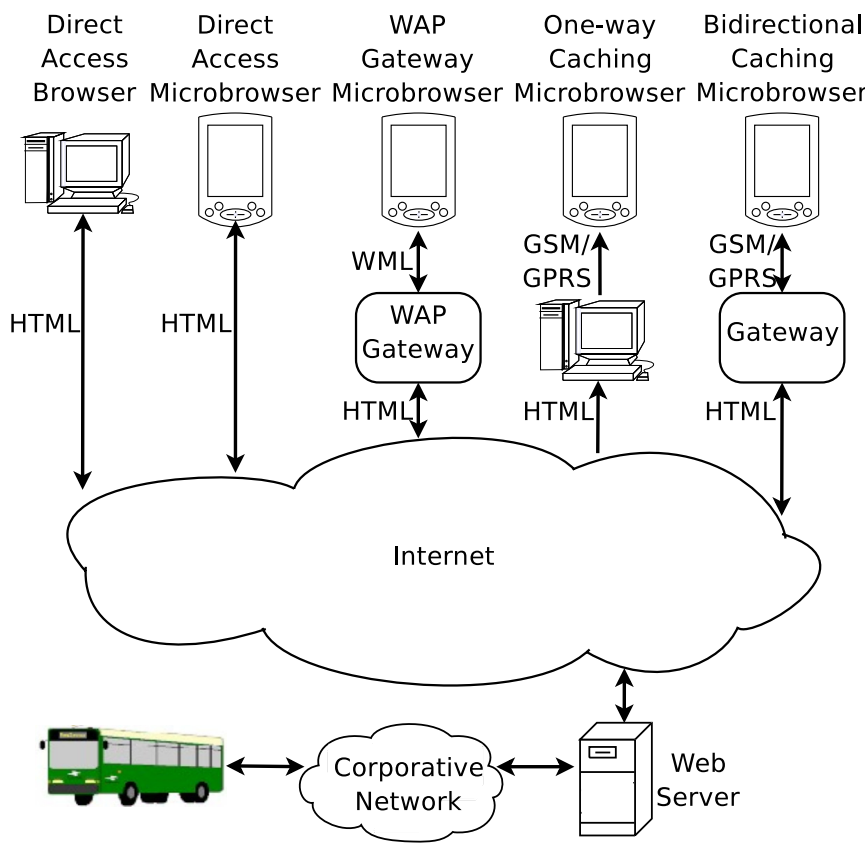

Figure 2: Passenger information system.

same agent platform. We have chosen to keep the mailbox in its home system because that frees us from the cost of mailbox tracking and it is simpler from the point of view of design. Moreover, generally, NM mode works well with small and medium systems with a few mobile agents and this is our case.

4.1.2.2 Message delivery The messages to the mobile agent must be sent first to the mailbox and then the messages must be delivered from the mailbox to the mobile agent. To achieve the second communication step, we can chose between two alternative methods: push operation $(P S)$ or pull operation $(P L)$. We have selected the push method, that is, the mailbox forwards messages to its mobile agent as they are received, for two reasons: firstly, frequently we need that the information arrives to the mobile information system on the buses as soon as possible, and, secondly, we prefer avoiding the overhead that would result of constant pull operations.

4.1.2.3 Synchronization between migration and delivery The migration of mailboxes and mobile agents can produce some message loss, if we do not take some precautions. To avoid them, we can perform some synchronization between the sending element and the receiving element-that is, between the sending host and the mailbox or between the mailbox and its mobile agent. The possible synchronization combinations are: no synchronization (NS), synchronization 
between host and mailbox (SHM), synchronization between mailbox and agent (SMA), and full synchronization (FS). Our system uses SMA synchronization to avoid message loss and to allow real-time delivering. As the mailbox does not migrate we do not need to synchronize it with the host sending the message, so SHM and FS are not applicable.

\subsubsection{Middleware}

The intent of the middleware is to integrate the on-board mobile systems into the overall corporative network. We have already described the various available communication media in subsection 4.1.2. We use those network infrastructures to achieve the integration of the different systems, taking into account efficiency and economy criteria.

A variety of information is used about the available resources: technical information about the network, rules of political nature imposed by enterprise management and geographical position information supplied by the GPS. This information about the available infrastructure is merged with the requirementsthat is, the quality of service-requested by the user of the middleware in its operation. Those requirements can include information like expiry date, reliability needs, and priority. All this information allows the middleware to automatically make decisions to perform its functionality taking into account its criterion of efficiency and economy.

\subsubsection{System software}

Next, we describe the main characteristics of the developed elements for the information systems aboard and how they use the middleware to transmit information to other systems. We have concentrated on obtaining scalable and flexible products, able to include new functionalities or services on the basis of subsequent technological innovations. Some examples of those possible onboard systems innovations are the incorporation of new payment systems and the redefinition of events management where performed actions could be of different nature. Besides, the middleware could be enriched with some new communication service offered by operators. In this case, the transmission will transparently use the more suitable medium, according to the updated infrastructure information and to the quality of service established by the middleware user, but without needing it to be aware of the communication infrastructure changing.

We have selected GNU/Linux as our development platform and to be the operating system used by the on-board equipments in production. To produce the software we have followed an object oriented development model with an abundant use of the client-server paradigm. The flexibility and openness has been also basic objectives among products main characteristics.

Finally, we have created an environment where the user can modify the functioning of the different elements through the use of tools with friendly user interfaces. 
The events manager is an example of this simplicity. It can generically handle different events or remarkable situations, programming its detection and the action to perform when the event takes place. This event manager can be easily configured - while it keeps running — by expressing in a declarative language what to detect and what to do when the event is detected. This declarative language uses a dictionary of terms containing the entities that can be monitored to detect event conditions on them that can be also defined by the user depending on his needs. At figure 3 we can see an example of events definition, where for every line, the first part defines the condition to test and the second the action to carry out when this condition occurs.

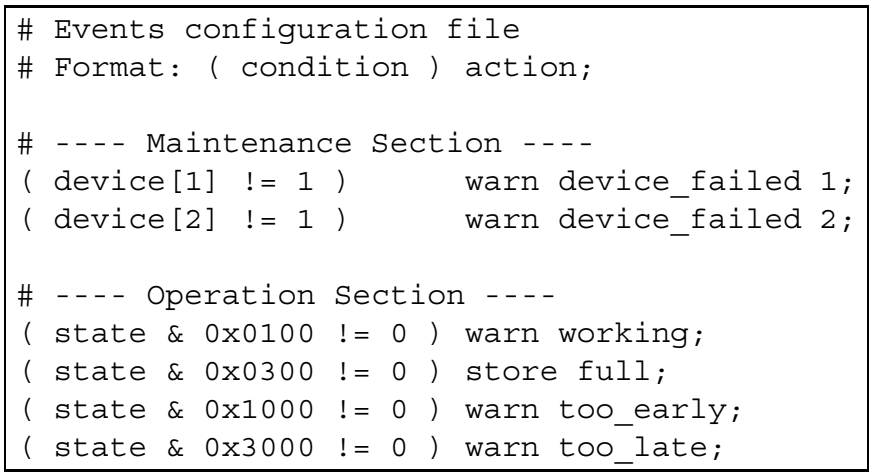

Figure 3: On-board system events configuration example.

\section{Conclusions}

We have described how technological advances can help a road public transport company to improve its service and undertake new challenges. Specifically, given the current state of information technologies, the pervasive computation model can be applied to build information systems with functionalities not proposed up to the moment. Basically, this model permits us to appropriately integrate mobile on-board information systems into the enterprise network vehicles and to offer high quality services to the public transport user. Besides, this model will allow us to develop flexible and scalable computation environments at an attractive cost. Those statements are supported by the results obtained from the system that we have developed. It is based on the pervasive computation paradigm and is currently working at the Global Salcai-Utinsa, S.A. Company. This enterprise has a fleet of 300 buses, transports 25 million passengers every year and carries out more than 4000 expeditions every day. 


\section{Acknowledgements}

We want to express our gratitude to all the organizations which have supported us in the execution of the projects that have given rise to the ideas that we have expounded in this article: the transport company Global Salcai-Utinsa, S.A., the Consejería de Economía y Hacienda del Gobierno Autónomo de Canarias and the Consejería de Turismo y Transportes del Gobierno Autónomo de Canarias.

\section{References}

[1] Weiser, M., The computer for the 21st century. Scientific American, 265(3), pp. 94-104, 1991.

[2] Saha, D. \& Mukherjee, A., Pervasive computing: A paradigm for the $21 \mathrm{st}$ century. 36(3), pp. 25-31, 2003.

[3] García, C., Alayón, F., Padrón, G., Rodríguez, M. \& Suárez, P., Architecture of an integral information system for public road transport of passengers. Urban Transport VI: Urban Transport and the Environment for the 21st Century, WIT Press, pp. 95-102, 2000. ISBN 1-85312-823-6.

[4] García, C., Alayón, F., Fernández, E. \& Medina, P., On board system model for road intelligent transport system. Urban Transport VII: Urban Transport and the Environment in the 21st Century, WIT Press, pp. 213-220, 2001. ISBN 1-85312-865-1.

[5] Kindberg, T. \& Fox, A., System software for ubiquitous computing. IEEE Pervasive Computing, 1(1), pp. 70-81, 2002.

[6] García, C. \& Alayón, F., A distributed system for mobile information communications. Management Information Systems 2004: Incorporating GIS and Remote Sensing, WIT Press, pp. 103-108, 2004. ISBN 1-85312-728-0.

[7] Cao, J., Feng, X., Lu, J. \& Das, S.K., Mailbox-based scheme for mobile agent communications. 35(9), pp. 54-60, 2002. 